\title{
Learning the DAG of Bayesian Belief Networks by Asking (Conditional) (In-)Dependence Questions
}

\author{
Claus Möbus \\ Learning and Cognitive Systems \\ Department of Computing Science \\ University of Oldenburg, D-26111 Germany \\ http://www.lks.uni-oldenburg.de/ \\ claus.moebus@uni-oldenburg.de
}

\author{
Hilke Garbe \\ Learning and Cognitive Systems \\ Department of Computing Science \\ University of Oldenburg, D-26111 Germany \\ http://www.lks.uni-oldenburg.de/ \\ hilke.garbe@uni-oldenburg.de
}

\begin{abstract}
Bayesian belief networks (BBNs) have become the de facto standard for the representation of uncertain knowledge. They consist of a qualitative and of a quantitative part describing the (in-)dependencies between the variables of interest as a directed acyclic graph (DAG) and the decomposition of the joint probability distribution (JPD) as a product of conditional probability distributions constrained by the structure of the DAG. In this paper we present a new constraint-based query procedure: Query-an-Oracle (QAO). We assume that an oracle - preferable a human domain expert - is at hand which is competent and willing to answer questions generated by QAO concerning the directed (causal) dependence and (conditional) independence of the relevant random variables in the domain. Compared to other structure learning methods (e.g. the $P C$ Algorithm of Peter Spirtes and Clark Glymour and the ICAlgorithm of Pearl) QAO has a number of advantages. It derives the DAG of the BBN with less computational complexity, with no redundant questions, and is able to exploit directed dependence information without urging oracles to differentiate between direct and indirect influence.
\end{abstract}

\section{Categories and Subject Descriptors}

I.2.4 Knowledge Representation Formalisms and Methods

\section{General Terms}

Algorithms, Measurement, Design, Experimentation, Human Factors

\section{LEARNING THE STRUCTURE OF BBNS}

We were looking for an alternative method which is formal, simple to use for domain experts, not restrictive in its assumptions, and not suffering from the same or similar drawbacks as the Bounded Strata Method [1] or the PC/IC-Algorithm [2, 3].

\section{The Query-an-Oracle (QAO) Algorithm}

This led to the development of a greedy knowledge acquisi-

\footnotetext{
Permission to make digital or hard copies of all or part of this work for personal or classroom use is granted without fee provided that copies are not made or distributed for profit or commercial advantage and that copies bear this notice and the full citation on the first page. To copy otherwise, or republish, to post on servers or to redistribute to lists, requires prior specific permission and/or a fee.

Copyright is held by the author/owner(s).

K-CAP'09, September 1-4, 2009, Redondo Beach, California, USA.

ACM 978-1-60558-658-8/09/09.
}

tion method for the construction of the transitive closure of the precedes/causes $(X, Y)$-relation[4,5].

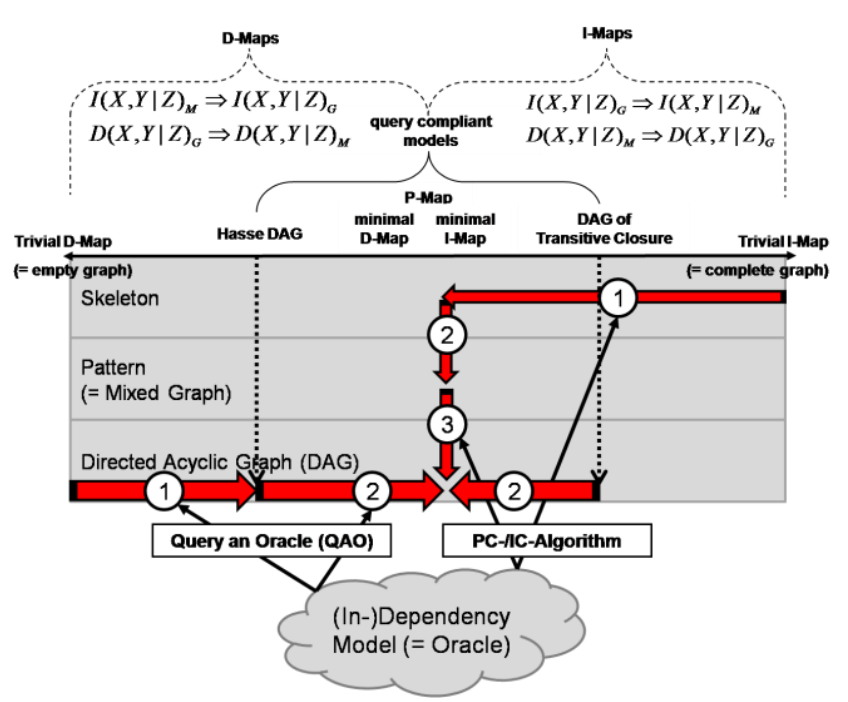

Figure 1. Model Space of Structure Learning Algorithms PCA, ICA, and QAO

First step of $Q A O$

In the first step of QAO this algorithm controls the nonredundant pair comparison of variables, and generates the Hasse and the transitive closure diagram of the partial-order relation precedes/causes $(\mathrm{X}, \mathrm{Y})$. The greedy behaviour is controlled by 13 production or propagation rules $[4,5]$. When a pair $(i, j)$ of variables is presented the oracle has to select a rating from a set of alternatives $\{i$ causes/precedes $j$, i follows $j$, $i$ neither causes/precedes nor follows $j\}$ internally abbreviated as $\{+(\mathrm{i}, \mathrm{j}),-(\mathrm{i}, \mathrm{j}), 0(\mathrm{i}, \mathrm{j})\}\}$. The QAO selects a special order of pair comparisons along the main diagonal of the adjacency matrix. When the variables are ancestral ordered this minimizes the number of pair comparisons queried from the oracle and maximizes the number of transitive inferences that are automatically generated by the algorithm. Because each transitive edge has to be confirmed by a (directional) conditional independence test in the second step of QAO the sequence of the query process is unimportant when we take the query complexity of both steps into account. Taking only the $+(\mathrm{i}, \mathrm{j})$ markings from the transitive closure we can reconstruct the Hasse diagram. 
MÖBUS, C. \& GARBE, H. Learning the DAG of Bayesian Belief Networks by Asking (Conditional) (In-)Dependence Questions, International Conference On Knowledge Capture (K-Cap 2009), Proceedings of the fifth international conference on Knowledge capture, 2009, p. 199-200, Redondo Beach, California, USA, ACM, New York, NY, USA, ISBN: 978-1-60558-658-8

\section{Second step of $Q A O$}

After termination of step 1 the semantics of the "+" edges has become unambiguous. The propagation process in step 1 denotes "+"-edges as not transitive. The semantics of the "+"edge between $\mathrm{X}$ and $\mathrm{Y}$ is that $X$ is a direct influencer of $Y$. In contrast to that the semantics of the "++"- edge is still ambiguous. The oracle has to decide whether "++"-edges denote a direct influencer or an indirect one. The true DAG (only known to nature) lies somewhere between the Hasse model and the transitive closure model (Fig. 1). In [4] we recommended a Markov blanket test for every variable for the decision in the second step. The improved solutions we propose here are much simpler. There are two alternative solutions for the second step.

(1) The first is a Markov blanket test only for “++"-edges.

(2) The other is a new kind of query to the oracle: Instead of the conventional non directional conditional independence I(X, Y | Z) QAO asks for each transitive " $++(\mathrm{X}, \mathrm{Y})$ "-edge in the transitive closure model a directional conditional independence $\mathrm{I}_{\mathrm{dir}}(\mathrm{Y}, \mathrm{X} \mid \mathrm{Z})$ : Does $X$ causally influence $Y$, when $Z$ is known? Yes or No?

\section{Complexity of $Q A O$}

A rough calculation of QAO's complexity for the first step gives following results. In the best case the true graph describes a total order. When we assume that the variables are ancestral ordered. QAO acquires the Hasse and the transitive closure diagrams by (n-1) questions only. Due to transitivity $(n-2)(n-1) / 2$ instances of a precedes/causes relations are inferred by QAO and need not be queried from the oracle. Thus, the first step of QAO has a best case complexity of $\mathrm{O}(\mathrm{n})$ for the query process and a computational complexity of $\mathrm{O}\left(\mathrm{n}^{4}\right)$ because the computation of transitive edges requires at most $[(\mathrm{n}-1)(\mathrm{n}-2) / 2]^{2}$ edge tests. In the worst case QAO needs $\mathrm{n}(\mathrm{n}-1) / 2$ ratings. Then the DAG has no transitivity edges and the second step is not required. In this case the complexity of the query process is $\mathrm{O}\left(\mathrm{n}^{2}\right)$ and the computational complexity for the transitivity calculation is $\mathrm{O}(\mathrm{c})$.

\section{A Comparison of PCA and QAO}

It is interesting to benchmark the improved QAO asking the directional conditional independence questions in the second step with the conventional constraint-based structure learning methods PCA [2] and ICA [3].

\section{Example 7.1.3 from Jensen and Nielsen}

The first example for a comparison is from [6, p.235f] (Figure 2). We wrote a computer program according to the Jensen and Nielsen's pseudo code. In the first step PCA generated 40 independence queries (10 independence queries $\mathrm{I}(\mathrm{X}, \mathrm{Y})$ of order zero, 21 queries $\mathrm{I}(\mathrm{X}, \mathrm{Y} \mid\{\mathrm{Z}\})$ of order one and 9 queries $\mathrm{I}(\mathrm{X}, \mathrm{Y} \mid\{\mathrm{Z} 1, \mathrm{Z} 2\})$ of order two). The independence queries I(A, B), I(B, C), I(C, D $\mid\{A\}), \mathrm{I}(\mathrm{A}, \mathrm{E} \mid$ $\{C, D\})$, and $\mathrm{I}(\mathrm{E}, \mathrm{B} \mid\{\mathrm{C}, \mathrm{D}\})$ were answered with "Yes". The result is the undirected skeleton graph (Figure 2). Then in the second step three production rules are applied to the skeleton. They mainly introduce directed v-structures (here two). Then in the third step the oracle is queried again to direct the remaining undirected edges. The total number of queries is 41 .

What is the behaviour of QAO? In the first step QAO asks 8 pair comparison queries. In the second step 2 directional conditional independence questions are generated $\mathrm{I}_{\text {dir }}(\mathrm{E}, \mathrm{A} \mid$ $\{C, D\})$ and $\mathrm{I}_{\mathrm{dir}}(\mathrm{E}, \mathrm{B} \mid\{\mathrm{C}, \mathrm{D}\})$ (Figure 2) and answered with "Yes". The total number of queries is 10 . Compared to PCA, this is a reduction of almost $75 \%$.
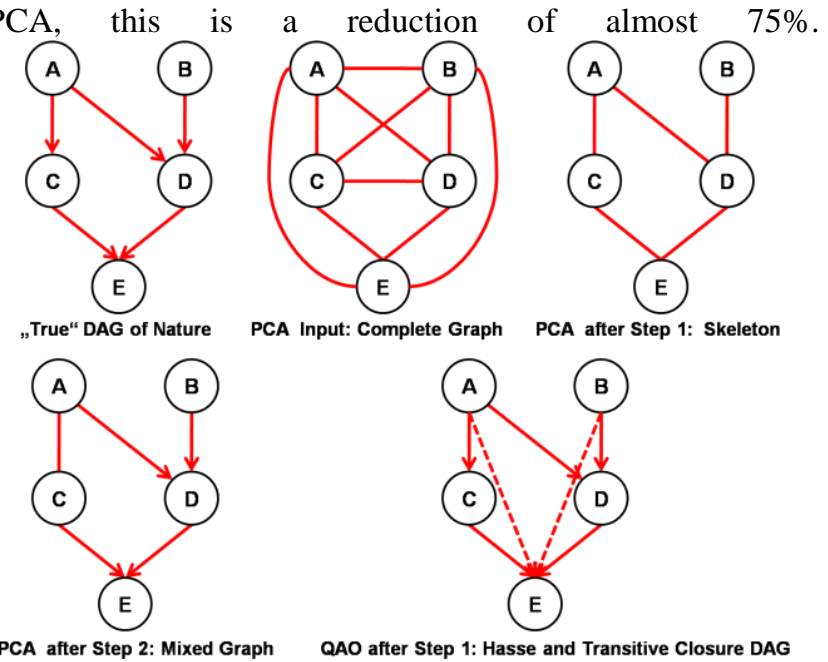

PCA after Step 2: Mixed Graph

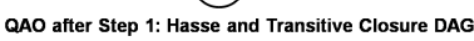

Figure 2. Steps 1 and 2 of PCA and Step 1 of QAO

\section{SUMMARY}

We presented a new structure learning algorithm Query-anOracle (QAO). This is in the second step an improved version of [4]. Compared to the well-known PC-algorithm of Spirtes et al. [2] and to the IC-algorithm of Pearl [3] it has a considerably smaller query complexity which is exactly $\mathrm{O}\left(\mathrm{n}^{2}\right)$. QAO is the first structure learning algorithm which exploits directional dependence ratings without urging oracles to give ratings of direct influence or direct control.

\section{REFERENCES}

[1] Pearl, J., 1998, Probabilistic Reasoning in Intelligent Systems, San Mateo, CA., ISBN 0-934613-73-7

[2] Spirtes, P., Glymour, C., and R. Scheines, Causation, Prediction, and Search, Camb, Mass.: MIT Press, 2000

[3] Pearl, J., 2001, Causality: Models, Reasoning, and Inference, Cambridge University Press

[4] Möbus, C., and Seebold, H., A Greedy Knowledge Acquisition Method for the Rapid Prototyping of Bayesian Belief Networks, in: Ch-K. Looi, G. McCalla, B. Bredeweg \& J. Breuker (eds), Artificial Intelligence in Education, Amsterdam: IOS Press, 2005, p. 875-877, ISBN 1-58603-530-4

[5] Möbus, C. \& Seebold, H., and Garbe, H., A Greedy Knowledge Acquisition Method for the Rapid Prototyping of Knowledge Structures, p.211-212, in: Clark, P.; Schreiber, G.; Proc. 3rd Int. Conf. on Knowledge Capture, 2005, NY: ACM Press, ISBN 1-59593-163-5;

[6] Jensen, F.V. \& Nielsen, Th. D, 2007, Bayesian Networks and Decision Graphs, Berlin: Springer 